\title{
Verschiedene Lagerungsmethoden und OP-Tisch-Modifikationen bei Schulteroperationen
}

\author{
Uwe Kleineidam, Hans Rieger
}

\section{Zusammenfassung}

Die stetig wachsende Zahl an Eingriffen an der Schulter (vorwiegend Arthroskopie) führt durch häufige Anwendung von unterschiedlichen Lagerungsmethoden und dem damit zusammenhängenden Problem am Ende zu einem Lagerungsstandard. Dieser unterstützt den Operateur bei der med. Versorgung und garantiert höchste Sicherheit für den Patienten.

Die Kenntnis im Umgang mit den in Anwendung gebrachten Lagerungs-Materialien sind ebenso wichtig wie die $\mathrm{Zu}$ sammenarbeit der an der Operation beteiligten Berufsgruppen.

In unserem Krankenhaus erfolgte die Entwicklung des Lagerungsstandards Schulteroperation in 3 Schritten.

1. Hüfttisch - normale OP-Tischplatte

2. Sonderzubehör Schulterplatte

3. Beach char Lagerung mit Schulterplatte
1. Normaler Tisch

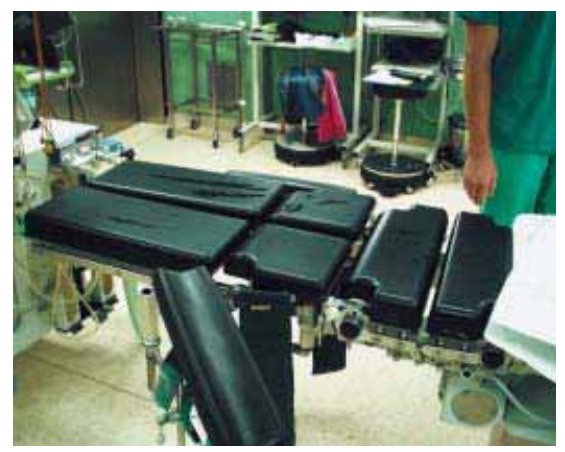

2. Sonderzubehör Schulterplatte

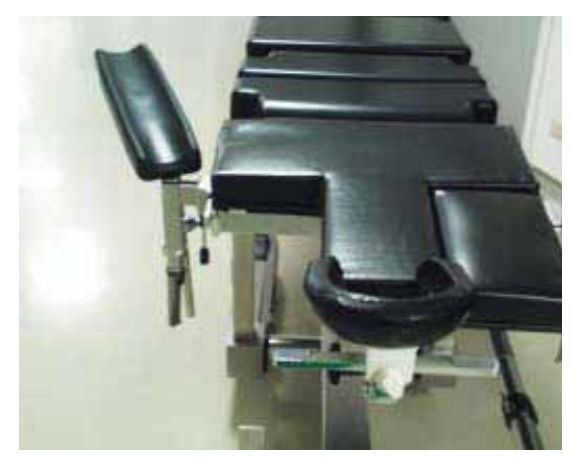

3. Beach char Lagerung mit Schulterplatte

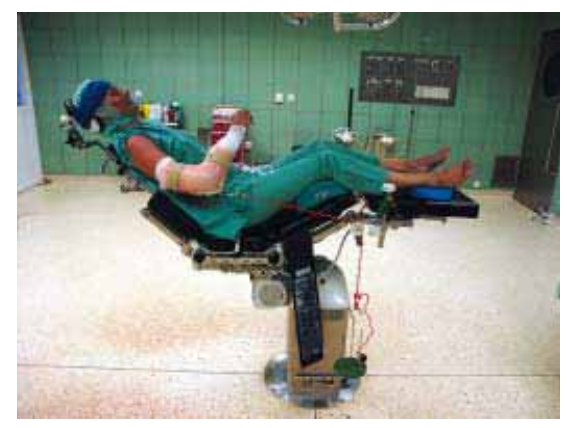

Zu 1: OP-Lagerung mit Normaltisch

Die Hauptschwierigkeiten bestanden darin, dass der Patient (Lagerung des Oberkörpers auf die Beinplatten) bei der endgültigen Lagerung recht instabil auf dem Tisch lag. Die Fixierung des Kopfes auf der Kopfschale gestaltete sich sehr aufwändig. Nur mit Sonderzubehör war dies schließlich dann doch sicher zu ermöglichen. Ein weiterer Nachteil bestand darin, dass nur wenige Mitarbeiter diese Lagerung durchführen konnten und oftmals deswegen darauf verzichtet wurde.

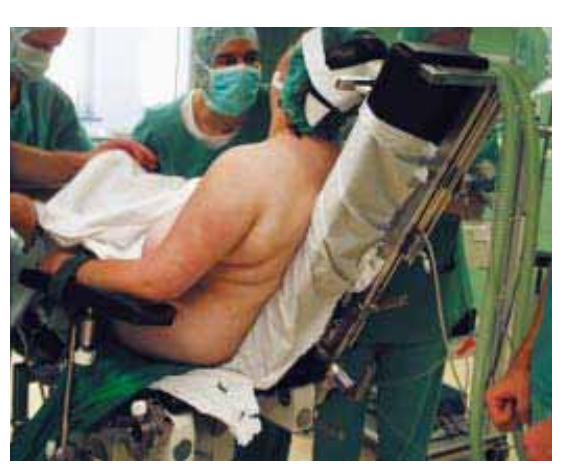

Da während der Lagerung permanent auf die Sicherheit des Patienten geachtet werden musste, waren viele Mitarbeiter in der Lagerungsphase gebunden.

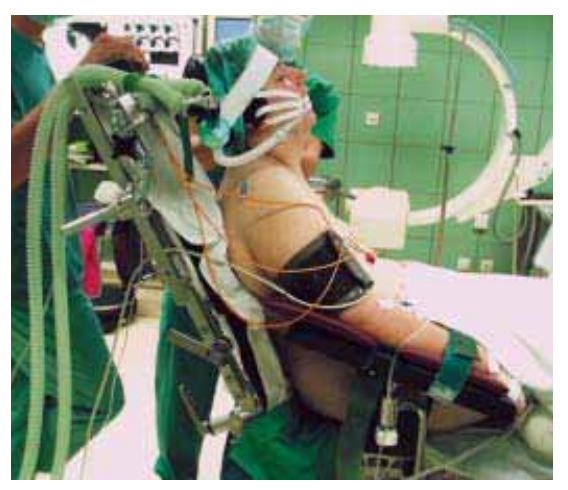

Eine Standardisierung war nur zum Teil möglich, da für jeden Patienten eine optimale Stellung und Positionierung der Schienen und Sonderzubehörteile angepasst werden musste.

Trotz aller Schwierigkeiten wurde diese Lagerung aber gerne eingesetzt, da sie während der Operationen einen guten Zugang zum Gelenk sicherstellte.
OP-JOURNAL 2002; 18: 262-266

(c) Georg Thieme Verlag Stuttgart . New York 


\section{Zu 2: Schulterplatte (Maquet)}

Eine deutliche Verbesserung wurde durch den Einsatz der neuen Oberkörperplatte (Sonderzubehör Maquet) erreicht. In Punkto Lagerungsstabilität war der Sicherheitsgewinn so groß, dass die bis dahin angewandte Lagerungsform praktisch nicht mehr zur Anwendung kam.

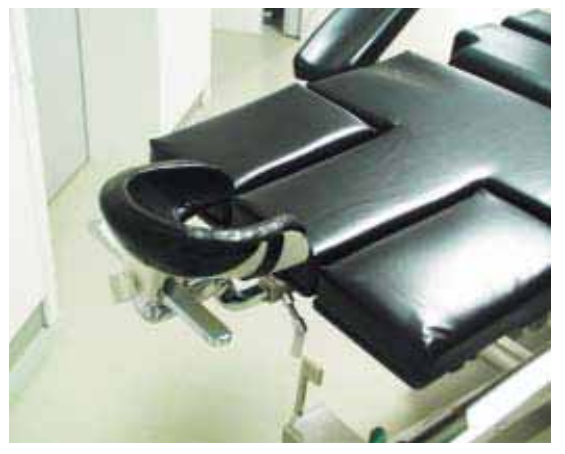

Der Patient ist schon ab der Patientenschleuse auf der endgültigen OP-Platte gelagert. Der personelle Aufwand, um einen freien Zugang zur Schulter zu erreichen, ist im Vergleich zur „alten“ Methode deutlich reduziert.

\section{Aufbau des Tisches mit Schulterplatte}

Der OP-Tisch befindet sich auf der OPTisch-Lafette. Der OP Tisch wird so umgebaut, dass beim Auffahren auf die OP Säule die lange Seite aus den Beinteilen, und die kurze, der Brustplatte, auf der Säulenmitte platziert ist (Hüfttisch): Abbau der Beinteile.

Achtung! Sicherungen und Paukenzüge zum Verstellen der Platte immer sorgfältig auf festen Halt und eingerasteten Sicherungsbolzen kontrollieren. Unfallgefahr!!!

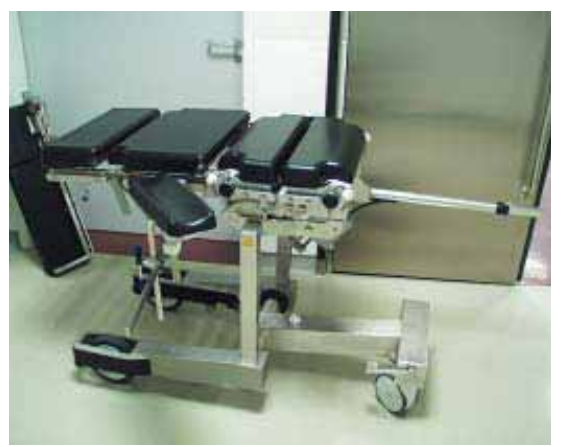

Abbau der Beinteile und Montage der Schulterplatte.
Einrasten der Sicherungsverschlüsse und wiederbefestigen der Paukenzüge.

Feste Schraubverbindungen der Schulterteilstücke kontrollieren. Bolzen und Verschluss der Schulterteile auf Funktion prüfen.

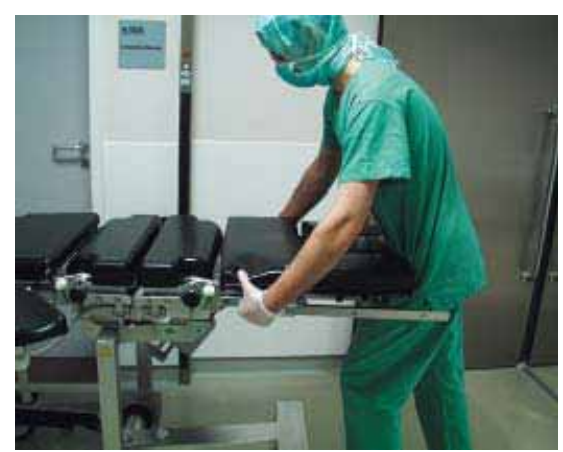

Schulterteile mit blauer Feststellschraube und kleinem Sicherheitsbolzen.
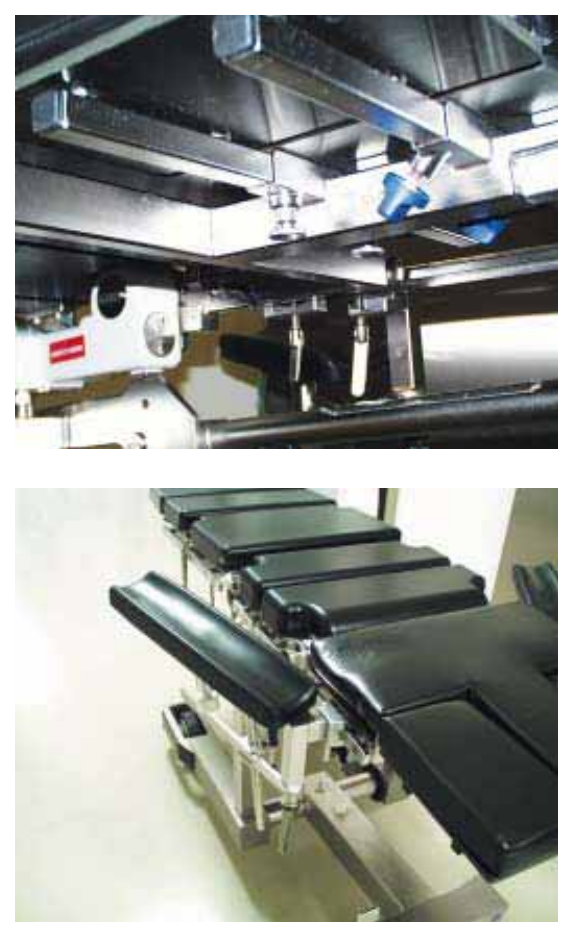

Anbau der Armlagerungsschienen auf beiden Seiten zur sicheren Lagerung und Fixierung der Arme.

\section{OP-Fotos (Schulterplatte)}

Bei größeren Patienten kann eine Beinverlängerung anmontiert werden. Die Kopfschale wird erst in Narkose montiert, und der Patient erst dann in die endgültige Position gebracht. Besondere Beachtung verdient die Lagerung des Kopfes.
Um eine stabile Lagerung in der Kopfschale über die gesamte Zeit der Operation zu gewährleisten, fixieren wir den Kopf mit einer selbstklebenden, elastischen Binde. Das enge Zusammenwirken mit der Anästhesie ist hierbei gefordert, da Beatmungsschläuche und sonstige Verkabelungen entsprechend der Operation verlegt und fixiert werden müssen.

Achtung! Keine Kompression der Augen durch Bindenfixierung.

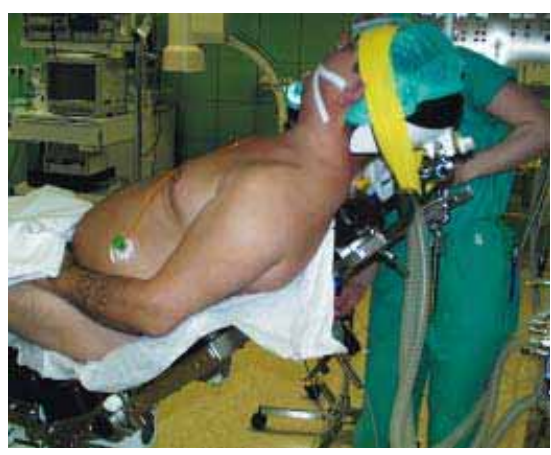

\section{Desinfektion}

Durch die völlige Freilagerung der zu operierenden Schulter, ist eine zirkuläre Desinfektion ohne weitere Probleme durchführbar.

Keine „Desinfektionsbäder“ sondern kontrollierbare Mengen verwenden.

Achtung! Desinfektionsmittel - Gefahr von Folgeschäden durch unkontrollierbare „Desinfektionsmittelpfützen“ gerade in Rücken und Gesäßbereich.

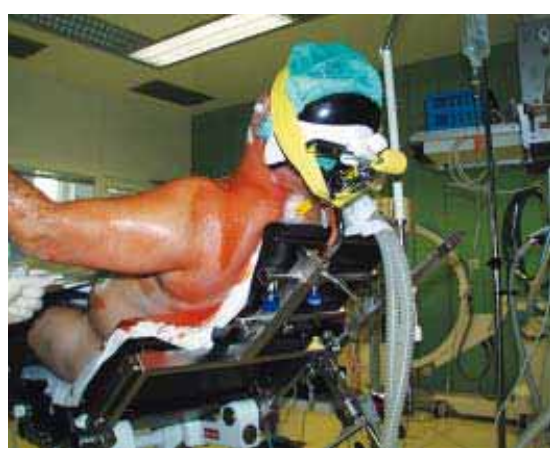

\section{Abdeckung}

Ob mit Textil- oder mit Papierabdeckungen eine sterile Abdeckung erfolgt, ist von Klinik zu Klinik verschieden. Wir decken mit Textiltüchern ab. Ein Abdeckstandard erleichtert die Abdeckmethode und wird 
mit entsprechender Übung von jedem Mitarbeiter sicher beherrscht.

1. Abdecken der Beine - Beintuch

Springer hält weiterhin den Arm

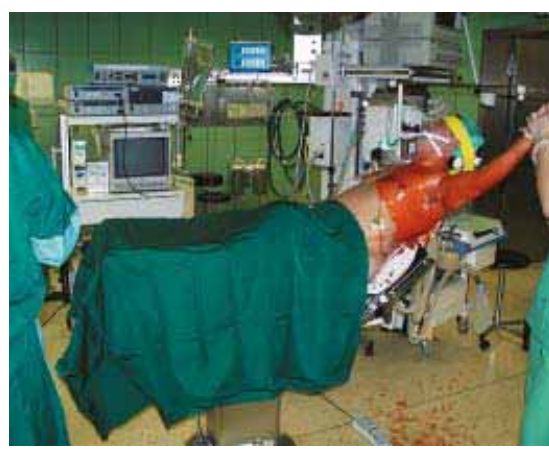

2. Übernahme des Armes mit einem sterilen Haltetuch. Überziehen der unsterilen Hand mit einem sterilen, Feuchtigkeit undurchlässigen Säckchen.

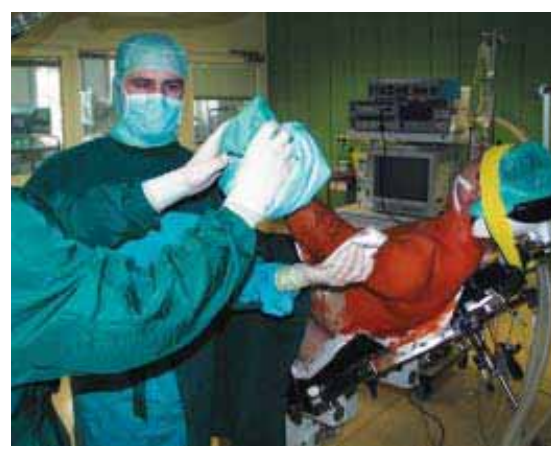

3. Fixieren des sterilen Handbeutels:

Die Fixierung muss einen sicheren Halt des sterilen Handsäckchens während der gesamten Operation gewährleisten.

Insbesondere bei der Arthroskopie im Schulterbereich kommen große Mengen an Spülflüssigkeit zum Einsatz.

Unserer Erfahrung nach hat sich die Fixation mit einer elastischen Binde, welche in 2 Touren angewickelt und verknotet wird, gut bewährt.

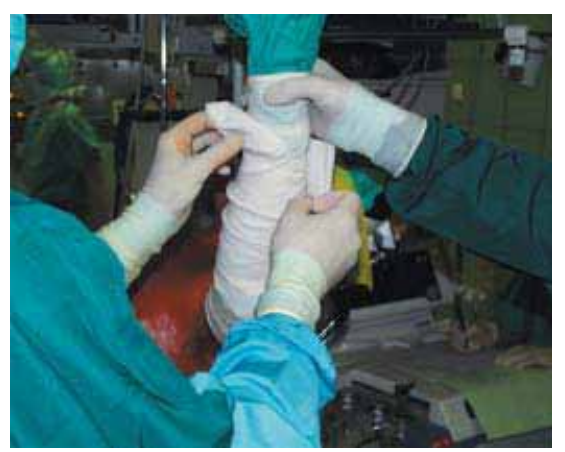

4. Anlegen eines U-Tuches - sterile Abdeckung der Extremität
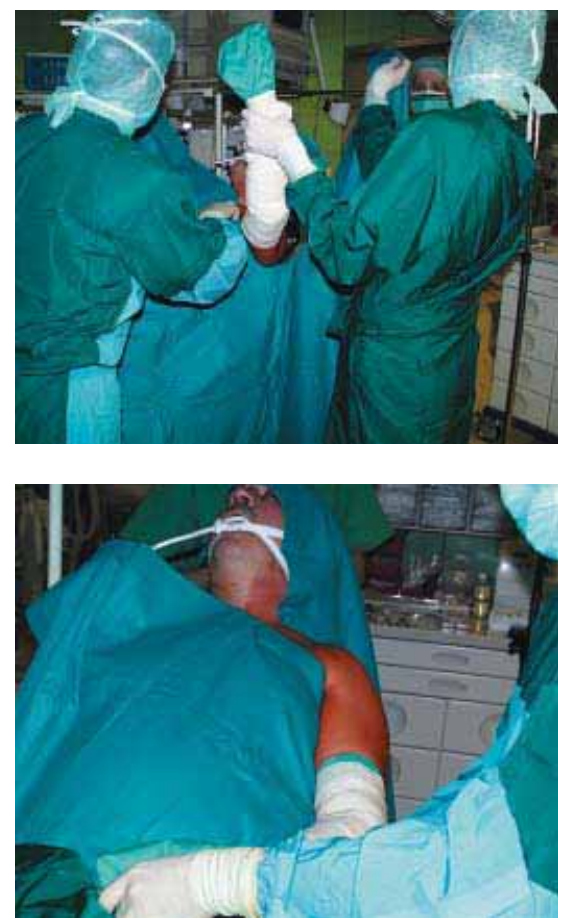

\section{Anästhesietuch}

Abschluss der Abdeckung. Fixieren des Tuches an Narkosebügel und 2 Infusionsständer.

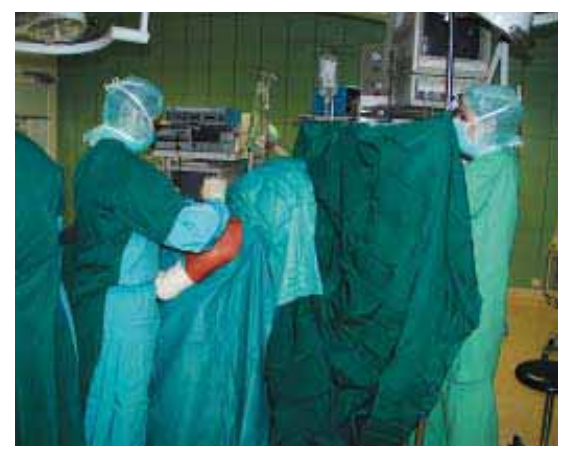

$\mathrm{Zu}$ 3: Beach char Lagerung mit Schulterplatte ( schematische Darstellung)

Basis ist der zum Hüfttisch umgebaute OP-Tisch mit Schulterplatte.

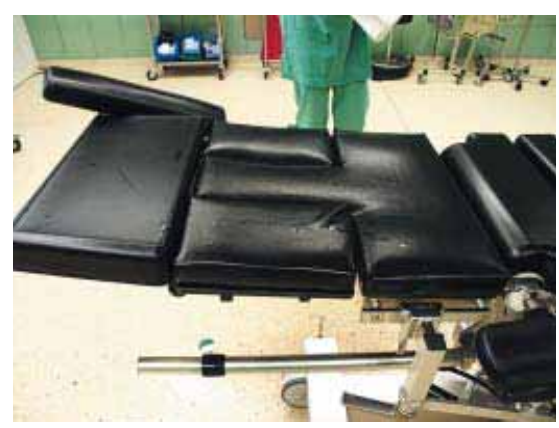

Entfernen des Schulterteiles

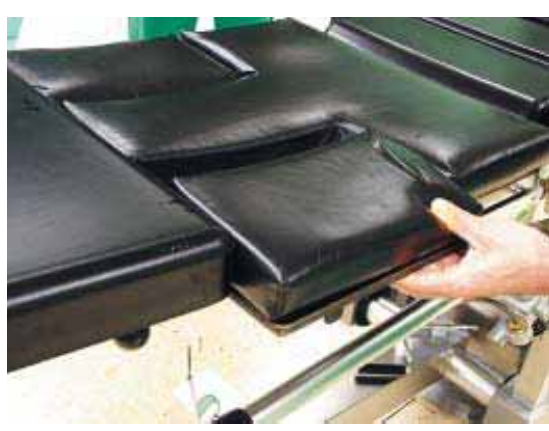

Anbau der Kopfschale

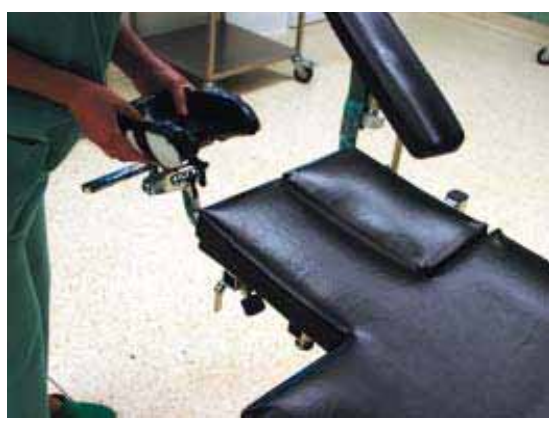

Gegebenenfalls Verlängerung des OP Tisches mit einer Beinverlängerung. Z.B. aus dem Zusatzlagerungsmaterial Extensionstisch.

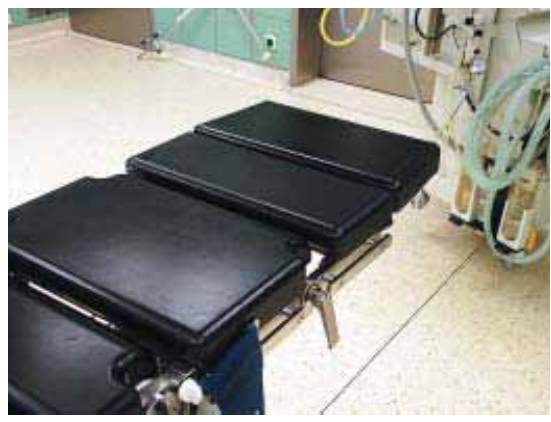

Anbringen einer Umlenkrolle zur Führung des Zugkabels

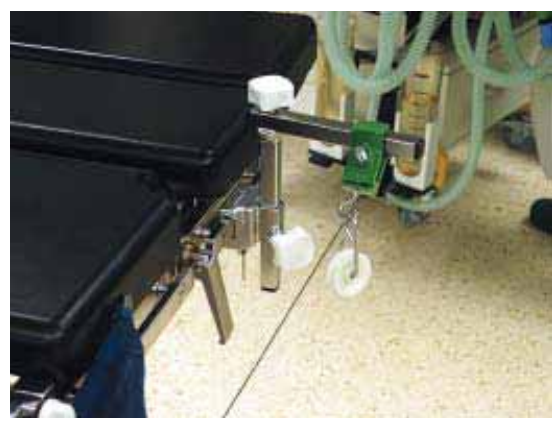

Bereitstellen eines Extensionsgewichtes ca. $1500 \mathrm{~g}$ zur leichten Extension des Armes, welcher in einer sterilen Kunststoffschiene gelagert wird. 
Kunststoffschiene in 2 unterschiedlichen Größen auf den Patienten anwendbar.

Resterilisierbar mit Gas.
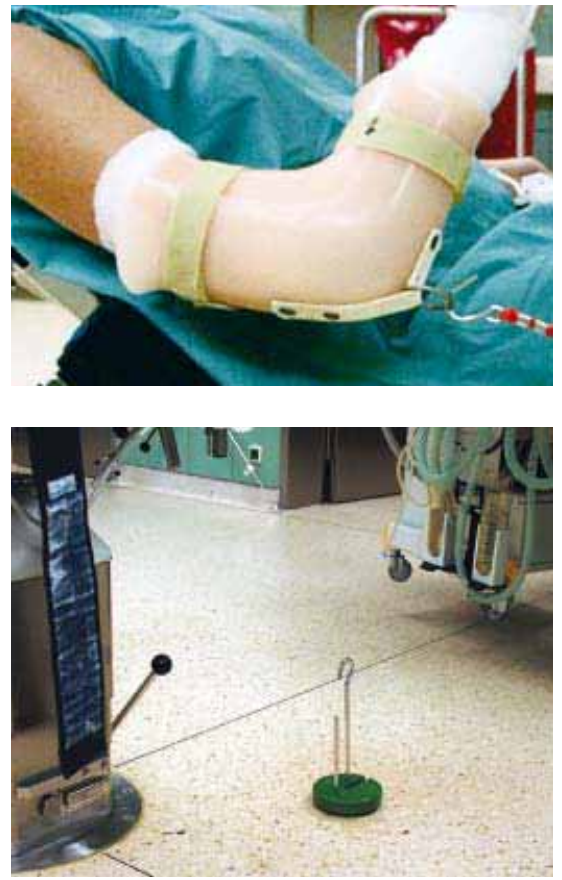

Anmontieren einer Armschiene an der Gegenseite mit Schienenverlängerung vom Extensionswagen.

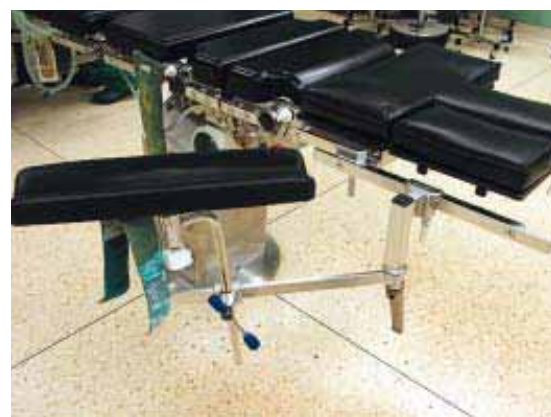

Fixieren des Kopfes mit einer selbstklebenden, elastischen Binde.

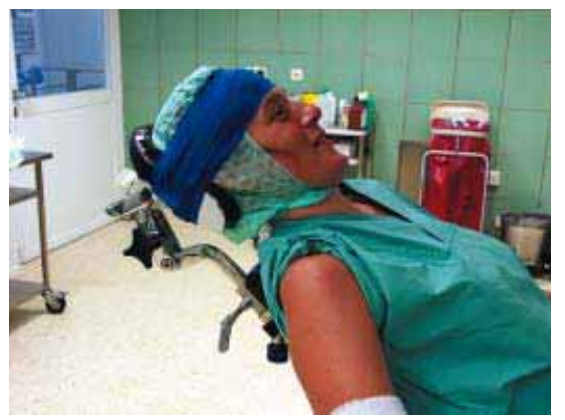

Oberkörper hoch lagern (in Absprache mit Anästhesie - Blutdruck!).

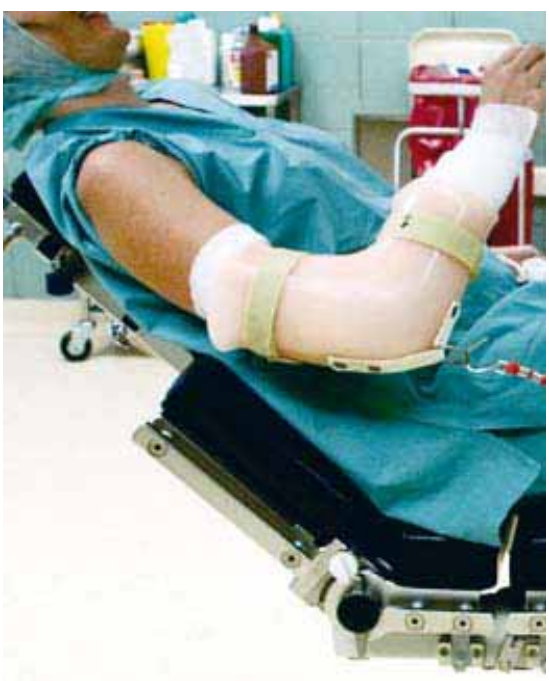

Beinteil anheben und in der Kniekehle mit einem halbrunden Polsterkissen unterstützen.

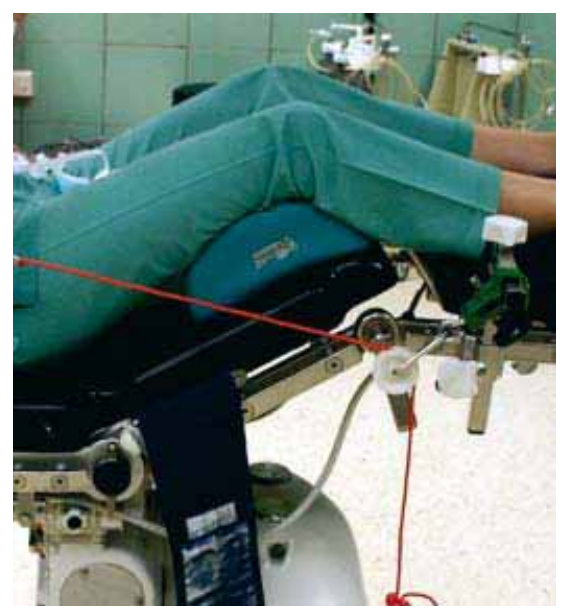

Abknicken des Fußteiles.

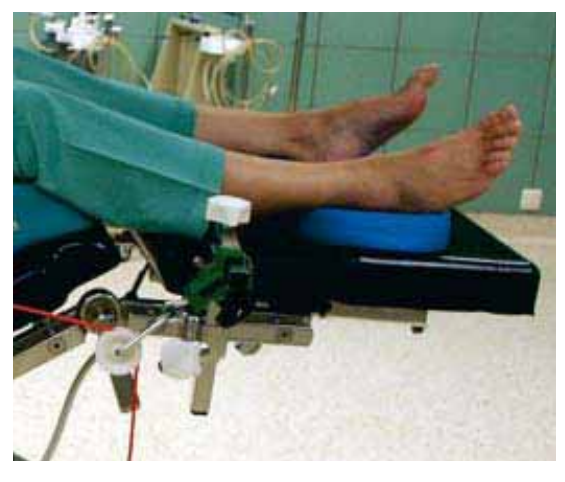

Unterpolsterung der Fersen mit Hohllagerungskissen.

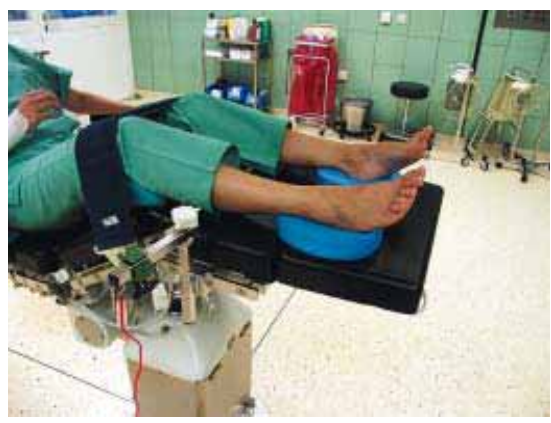

Sichere Lagerung des Armes der OP fernen Seite.

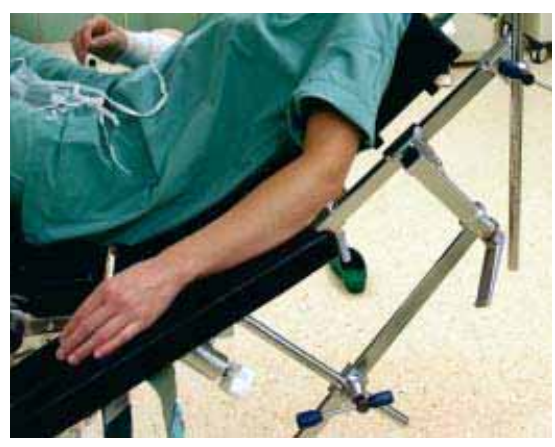

Positionierung des Narkosebügels.

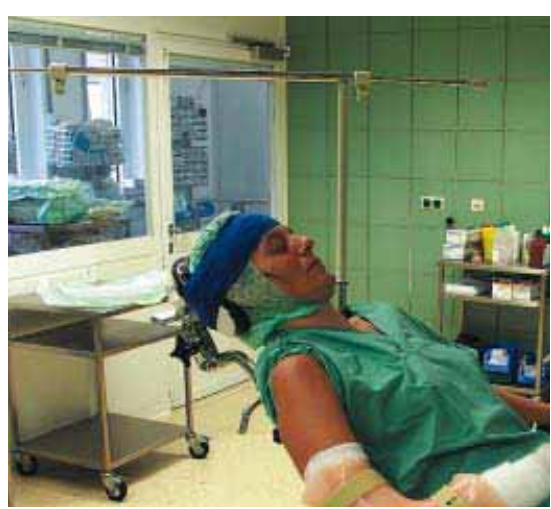

Fertige Lagerung mit eingehängter Extension der Armschiene und Zuggewicht $1500 \mathrm{~g}$.

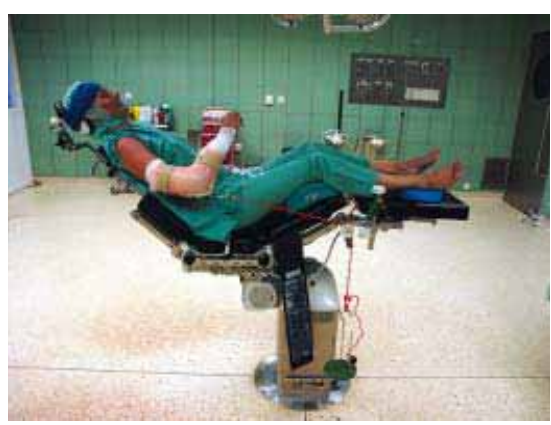




\section{Weitere Optimierung der Lagerung:}

Sonderzubehör zur Kopffixierung (Maquet).

Vorteil: Der Kopf muss nicht mehr mit einer elastischen Binde fixiert werden. Die Sicherheit der stabilen Kopflagerung ist perfekt gelöst. 2 zusätzliche Polster fixieren zusätzlich den Kopf am Schädeldach und im Stirnbereich.

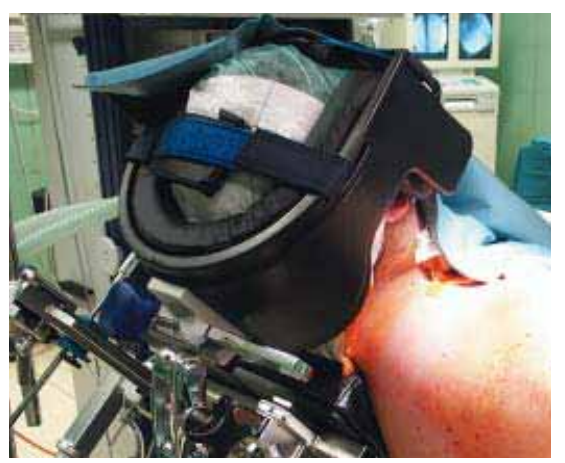

\section{Seitenansicht.}

Zusätzliche Fixierung mit einem Polster im Stirnbereich. Durch gute Polsterung ist eine zusätzliche Polsterung im Kopf und Nackenbereich nicht mehr notwendig.

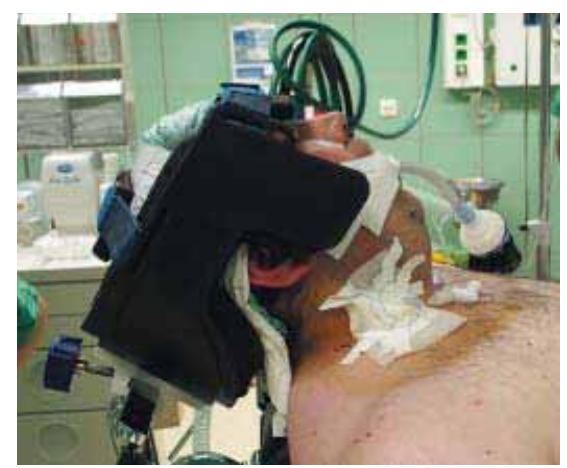

Fixierung der Kopfhalterung im Mittelloch der Schulterplatte.

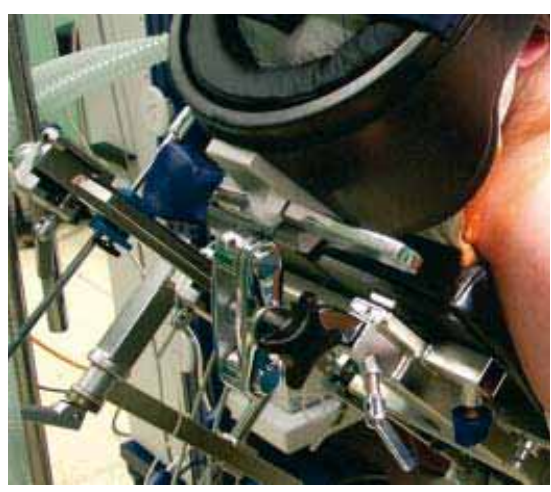

Uwe Kleineidam

OP-Leitung

(Text und Bilder)

Dr. med. Hans Rieger

Unfallchirurg

(med. Unterstützung)

Krankenhaus der Barmherzigen Brüder Prüfeninger Straße 86

93049 Regensburg 